\title{
Vector and Ordered Variational Inequalities and Applications to Order-Optimization Problems on Banach Lattices
}

\author{
Linsen Xie, ${ }^{1}$ Jinlu $\mathrm{Li}^{2}{ }^{2}$ and Wenshan Yang ${ }^{3}$ \\ ${ }^{1}$ Department of Mathematics, Lishui University, Lishui, Zhejiang 323000, China \\ ${ }^{2}$ Department of Mathematics, Shawnee State University, Portsmouth Ohio 45662, USA \\ ${ }^{3}$ Department of Mathematics, Zhejiang Normal University, Jinhua, Zhejiang 321000, China
}

Correspondence should be addressed to Jinlu Li; jli@shawnee.edu

Received 28 May 2013; Accepted 15 July 2013

Academic Editor: Shih-sen Chang

Copyright (C) 2013 Linsen Xie et al. This is an open access article distributed under the Creative Commons Attribution License, which permits unrestricted use, distribution, and reproduction in any medium, provided the original work is properly cited.

We investigate the connections between vector variational inequalities and ordered variational inequalities in finite dimensional real vector spaces. We also use some fixed point theorems to prove the solvability of ordered variational inequality problems and their application to some order-optimization problems on the Banach lattices.

\section{Introduction}

Let $X$ be a real Banach space with its norm dual $X^{\prime}$. Let $C$ be a nonempty convex subset of $X$ and $f: C \rightarrow X^{\prime}$ a single valued mapping. The variational inequality problem associated with $C$ and $f$, simply denoted as $\operatorname{VI}(C, f)$, is to find an $x^{*} \in C$ such that

$$
\left\langle f\left(x^{*}\right),\left(x-x^{*}\right)\right\rangle \geq 0, \quad \forall x \in C .
$$

The variational inequality problem $\operatorname{VI}(C, f)$ has been extensively studied by many authors. This theory has been widely applied to optimization theory, game theory, economic equilibrium, mechanics, and so forth. It has been recognized as an important branch in nonlinear analysis (see, e.g., [1-5]).

The theory of variational inequality was first extended by Giannessi [6] in 1980 to vector variational inequality problems in finite dimensional vector spaces. Since then, it has been deeply studied by many authors such as Giannessi [6-10], Agarwal et al. [11], Mastroeni and Pellegrini [12], Li and Huang [13], Luc [14], and Facchinei and Pang [15] and also has been applied to many fields such as vector optimization problems and vector equilibrium problems. In 2005, Huang and Fang [10] generalized the vector variational inequality problems from finite dimensional vector spaces to the Banach spaces. We recall the extension as follows.
For any positive integer $n$, let $\mathbb{R}^{n}$ denote the $n$ dimensional Euclidean space. Let $C$ be a convex and pointed cone of $\mathbb{R}^{n}$ and let $\succcurlyeq_{c}$ be the partial order on $\mathbb{R}^{n}$ induced by $C$. Let $f: \mathbb{R}^{m} \rightarrow \mathbb{R}^{n \times m}$ be a matrix-valued function. Let $K$ be a closed convex subset of $\mathbb{R}^{m}$. The vector variational inequality problem associated with $f, C$, and $K$ is to find an $x^{*} \in K$ such that

$$
f\left(x^{*}\right)\left(y-x^{*}\right) \kappa_{c} 0, \quad \forall y \in K
$$

As a special case, one may consider finding an $x^{*} \in K$ such that

$$
f\left(x^{*}\right)\left(y-x^{*}\right) \succcurlyeq_{c} 0, \quad \forall y \in K .
$$

In some economics circumstances, the preferences of a certain type of outcomes may be described by a special partial order, in particular a lattice order, on the space of outcomes. In this case, any preference inequalities and optimal problems must be defined under the given partial order that describes the preferences. Based on this motivation, Li and Wen [16] recently extended the variational inequality problem (1) to the following case: let $X$ be a Banach space and let $\left(U ; \geqslant^{U}\right)$ be a Banach lattice, where $X$ is considered as the income domain and $\left(U ; \geqslant^{U}\right)$ is considered as the production outcome space. Let $K$ be a nonempty closed convex subset of $X$ and let $F$ be a mapping from $K$ to $L(X, U)$. Then the ordered variational 
inequality problem associated with $K, F$, and $U$, denoted by $\operatorname{VOI}(K, F, U)$, is to find an $x^{*} \in K$ such that

$$
F\left(x^{*}\right)\left(x-x^{*}\right) \geqslant 0, \quad \forall x \in K .
$$

More general, similar to problem (2) is to find an $x^{*} \in K$ such that

$$
F\left(x^{*}\right)\left(y-x^{*}\right) \aleph^{U} 0, \quad \forall y \in K .
$$

From the Choquet-Kendall theorem, for a given convex and pointed cone $C$ in the $n$-dimensional Euclidean space $\mathbb{R}^{n}$, the partial order $\succcurlyeq_{c}$ on $\mathbb{R}^{n}$ induced by $C$ may not be a lattice order on $\mathbb{R}^{n}$. Furthermore, we can provide counterexamples to show that even in the case that $\succcurlyeq_{c}$ on $\mathbb{R}^{n}$ induced by $C$ is a lattice order on $\mathbb{R}^{n},\left(\mathbb{R}^{n}, \succcurlyeq_{c}\right)$ may not be a Banach lattice. Hence problems (5) and (4) are generalizations of (2) and (3), respectively, only if $\left(\mathbb{R}^{n}, \geqslant_{c}\right)$ is considered as a Banach lattice. In this paper, we investigate the connections between problems (2), (3) and problems (4), (5).

In [16], Li and Wen proved some solvability theorems about the ordered variational inequality problems (4) and (5) by applying the KKM mappings and the Fan-KKM theorem. In this paper, we use some fixed point theorem to prove the solvability of the ordered variational inequality problems (4) and (5) and the existence of solutions to some orderoptimization problems in the Banach lattices.

\section{Preliminaries}

In this section, we recall some concepts and properties of the Banach lattices. These properties will be frequently used throughout this paper. For more details, the readers are referred to [17]. We also recall the concept of vector variational inequality problems in the $n$-dimensional Euclidean space $\mathbb{R}^{n}$ and the variational order inequality problems in the Banach lattices.

A Banach space $X$ equipped with a lattice order $\succcurlyeq^{X}$ is called a Banach lattice, which is written as $\left(X ; \geqslant^{X}\right)$, if the following properties hold:

(b1) $x \geqslant^{X} y$ implies $x+z \geqslant^{X} y+z$, for all $x, y, z \in X$,

(b2) $x \geqslant^{X} y$ implies $\alpha x \geqslant^{X} \alpha y$, for all $x, y \in X$ and $\alpha \geq 0$,

(b3) $|x| \geqslant X|y|$ implies $\|x\| \geq\|y\|$, for every $x, y \in X$,

where, as usual, the origin of $X$ is denoted by $0, x^{+}=x \vee 0$, $x^{-}=(-x) \vee 0$, and $|x|=x^{+} \vee x^{-}$, for all $x \in X$, and $\|\cdot\|$ denotes the norm of the Banach space $X$. Let $\left(X ; \geqslant^{X}\right)$ be a Banach lattice. The positive cone of $\left(X ; \geqslant^{X}\right)$, denoted by $X^{+}$, is the subset $\left\{x \in X: x \geqslant{ }^{X} 0\right\}$. Analogously, we define the negative cone of $\left(X ; \succcurlyeq^{X}\right)$, denoted by $X^{-}$, as the subset $\{x \in X$ : $\left.x \preccurlyeq^{X} 0\right\}$. Then the properties of the lattice order $\geqslant^{X}$ in $\left(X ; \geqslant^{X}\right)$ immediately imply

$$
X^{-}=-X^{+}, \quad X^{+} \cap X^{-}=\{0\} .
$$

From Section 4.1 in [17], we see that every normed Riesz space $\left(X, \geqslant^{X}\right)$ is a special example of locally convex-solid Riesz spaces. Meanwhile, the Banach lattices are normed Riesz spaces; therefore, as a special case of Theorem 3.46 [17], we have the following closeness properties for the positive cones of the Banach lattices, which are very useful in the contents of this paper.

Theorem 1 (see $[16,17])$. Both of the positive and negative cones of any Banach lattice are norm closed, so they are weakly closed.

Lemma 2 (see [16]). Let $\left(X ; \geqslant^{X}\right)$ be an arbitrary Banach lattice. Then both of the positive and negative cones $X^{+}$and $X$ are order-closed. That is, for any net $\left\{x_{\alpha}\right\}$ in $X^{+}$(or in $X^{-}$), which order-converges to $x, x \in X^{+}$(or in $X^{-}$).

For any pair of elements $x$ and $y$ in a Banach lattice $\left(X ; \succcurlyeq^{X}\right)$, we say that $x \succ^{X} y$ whenever $x \geqslant^{X} y$ and $y \neq^{X} x$. Define the strictly positive and the strictly negative cones of the Banach lattice $\left(X ; \succcurlyeq^{X}\right)$, respectively, by

$$
X^{++}=\left\{x \in X: x>^{X} 0\right\}, \quad X^{--}=\left\{x \in X: x<{ }^{X} 0\right\} .
$$

These two cones possess the following properties:

$$
X^{--}=-X^{++}, \quad X^{++} \cap X^{--}=\phi .
$$

In general, both of $X^{++}$and $X^{--}$are neither closed nor open in $X$. This can be demonstrated by the following example.

Example 3. Let $\left(\mathbb{R}^{2}, \geqslant^{2}\right)$ be the Hilbert lattice with the coordinate lattice order $\geqslant^{2}$ on $\mathbb{R}^{2}$; that is, for any pair of points $\left(x_{1}, y_{1}\right),\left(x_{2}, y_{2}\right) \in \mathbb{R}^{2},\left(x_{1}, y_{1}\right) \geqslant^{2}\left(x_{2}, y_{2}\right)$ if and only if $x_{1} \geq x_{2}$ and $y_{1} \geq y_{2}$. The positive cone $\left(\mathbb{R}^{2}\right)^{+}$of $\left(\mathbb{R}^{2}, \geqslant^{2}\right)$ is the set $\left\{(x, y) \in \mathbb{R}^{2} \mid x \geq 0\right.$ and $\left.y \geq 0\right\}$, which is closed in $\mathbb{R}^{2}$. The strictly positive cone $\left(\mathbb{R}^{2}\right)^{++}$of $\left(\mathbb{R}^{2}, \geq^{2}\right)$ is the set $\left\{(x, y) \in \mathbb{R}^{2} \mid x \geq 0\right.$ and $\left.y \geq 0\right\} \backslash\{(0,0)\}$, which is neither closed nor open in $\mathbb{R}^{2}$. Similar to $\left(\mathbb{R}^{2}\right)^{++}$, the strictly negative cone $\left(\mathbb{R}^{2}\right)^{--}$of $\left(\mathbb{R}^{2}, \geqslant^{2}\right)$ is the set $\left\{(x, y) \in \mathbb{R}^{2} \mid x \leq 0\right.$ and $y \leq 0\} \backslash\{(0,0)\}$, which is neither closed nor open in $\mathbb{R}^{2}$.

Any two elements $x$ and $y$ in a Banach lattice $\left(X ; \geqslant^{X}\right)$ are said to be noncomparable under the order $\geqslant^{X}$, if neither $x \geqslant{ }^{X} y$ nor $x \geqslant{ }^{X} y$ holds, which is denoted by $x \ltimes^{X} y$. The nonzero-comparable set of a Banach lattice $\left(X ; \geqslant^{X}\right)$, denoted by $X^{\bowtie}$, is the subset $\left\{x \in X: x \bowtie^{X} 0\right\}$.

From Theorem 3.46 [17] and Lemma 2.3 [16] recalled in this section, the union of the positive and negative cones of a Banach lattice is norm closed. It immediately implies the following lemma.

Lemma 4. The nonzero-comparable set $X^{\bowtie}$ of a Banach lattice $\left(X ; \succcurlyeq^{X}\right)$ is open in $X$.

Proof. It is clear that $X^{\bowtie}=X-\left(X^{+} \cup X^{-}\right)$. Then the lemma follows from that both $X^{+}$and $X^{-}$are closed.

Definition 5. Let $X$ be a Banach space and let $\left(U ; \geqslant^{U}\right)$ be a Banach lattice. Let $C$ be a nonempty convex subset of $X$ and 
$F: C \rightarrow L(X, U)$ a mapping, where $L(X, U)$ denotes the Banach space of continuous linear operators from $X$ to $U$. The ordered variational inequality problem associated with $C, F$, and $U$, denoted by $\operatorname{VOI}(C, F, U)$, is to find an $x^{*} \in C$ such that

$$
F\left(x^{*}\right)\left(x-x^{*}\right) \geqslant 0, \quad \forall x \in C,
$$

where, as usual, 0 denotes the origin of $U$. If $f$ is linear, then the problem $\operatorname{VOI}(C, F, U)$ is called a linear ordered variational inequality problem; otherwise, it is called a nonlinear ordered variational inequality problem.

Many authors study the solvability of variational inequalities by applying the Fan-KKM theorem and fixed point theorems (see [18]). In [16], Li and Wen used the FanKKM theorem to prove the existence of solutions for some ordered variational inequalities. In this paper, we use fixed point theorems to show the solvability of some ordered variational inequalities. We first recall the concept of upper semicontinuous mappings from some topological spaces to topological spaces.

Definition 6 (see [11]). Let $X, Y$ be Hausdorff topological spaces. Let $F: X \rightarrow 2^{Y} \backslash\{\phi\}$ be a set valued mapping. For a point $x_{0} \in X$, if, for any neighborhood $\delta_{Y}\left(F\left(x_{0}\right)\right)$ of the set $F\left(x_{0}\right)$ in $Y$, there exists a neighborhood $\delta_{X}\left(x_{0}\right)$ of the point $x_{0}$ in $X$, such that

$$
F(x) \subset \delta_{Y}\left(F\left(x_{0}\right)\right), \quad \forall x \in \delta_{X}\left(x_{0}\right),
$$

then $F$ is said to be upper semicontinuous at point $x_{0}$. If $F$ is upper semicontinuous at every point in $X$, then $F$ is said to be upper semicontinuous on $X$.

The following theorem provides a criterion to check if a mapping is upper semi-continuous, which is useful in the following contents (see Agarwal et al. [11], for details).

Theorem 7 (see [11]). Let $X, Y$ be Hausdorff topological spaces with $Y$ compact. Let $F: X \rightarrow 2^{Y} \backslash\{\phi\}$ be a set valued mapping. If $F$ has a closed graph, then $F$ is upper semi-continuous on $X$.

Bohnenblust-Karlin Fixed Point Theorem (1950). Let $X$ be a Banach space and $K$ a nonempty compact convex subset of $X$. Let $T: K \rightarrow K$ be an upper semi-continuous mapping with closed convex values. Then $T$ has a fixed point.

\section{Connections between Vector Variational Inequalities and Ordered Variational Inequalities}

In finite dimensional real vector spaces, the vector variational inequalities defined by (2) and (3) and the ordered variational inequalities defined in (9) are all generalizations of variational inequalities defined in (1). In this section, we investigate the connections between these generalizations. We first recall a useful type of partial order on a vector space that is induced by a cone in this space.
Let $C$ be a nonempty subset of a topological linear space $X$. $C$ is called a (pointed) cone in $X$ if it satisfies the following two conditions:

(1) $C \neq\{0\}$ and $a C \subseteq C$, for any nonnegative number $a$;

(2) $(-C) \cap C=\{0\}$.

Let $C$ be a closed convex cone in a topological linear space $X$. The partial order $\succcurlyeq_{C}$ on $X$ induced by $C$ is defined as follows:

$$
x \geqslant_{C} y \quad \text { if and only if } x-y \in C .
$$

Hence, the topological linear space $X$ equipped with the partial order $\geqslant_{C}$ is a partially ordered topological linear space, which is denoted by $\left(X, \geqslant_{C}\right)$ and is said to be induced by the cone $C$.

It is important to notice that the partial order $\succcurlyeq_{C}$ on the partially ordered topological linear space $\left(X, \geqslant_{C}\right)$ is far away from being a lattice order; that is, the partially ordered topological linear space $\left(X, \succcurlyeq_{C}\right)$ may not be a Riesz space (vector lattice). This can be demonstrated by the following simple example.

Example 8. In $\mathbb{R}^{3}$, take $C$ to be the closed convex cone as follows:

$$
C=\left\{(x, y, 0) \in \mathbb{R}^{3}: x \geq 0, y \geq 0\right\} .
$$

We claim that the partial order $\succcurlyeq_{C}$ on $\mathbb{R}^{3}$ is not a lattice order. In fact, we take two points $a=(1,2,1)$ and $b=(2,1,2)$. One can see that the subset $\{a, b\}$ has no upper bound in $\left(\mathbb{R}^{3}, \succcurlyeq_{C}\right)$. It immediately implies that $a \vee b$ does not exist. Hence $\succcurlyeq_{C}$ is not a lattice order on $\mathbb{R}^{3}$ and $\left(\mathbb{R}^{3}, \geqslant_{C}\right)$ is not a vector lattice.

Here we consider some special cones in real vector spaces such that their induced partial order is a lattice order. For more details, the reader is referred to Kendall [19].

Let $B$ be a nonempty convex set in a real vector space $X$. The subset $\{\lambda a+(1-\lambda) b: \lambda$ real; $a, b \in B\}$ of $X$ is called the minimal affine extension of $B$. Suppose that the minimal affine extension of $B$ does not contain the zero vector. Let $C=$ $\{\lambda b: \lambda \geqslant 0, b \in B\}$. Then $C$ is a pointed convex cone in $X$.

Kinderlehrer and Stampacchia [4] calls a nonempty convex set $S$ in a real vector space $X$ a simplex when the intersection of two positively homothetic images of $S$,

$$
x+\lambda S, \quad y+\mu S \quad(x, y \in X ; \lambda, \mu \geqslant 0),
$$

is empty or is a set $a+\lambda S(a \in X, v \geqslant 0)$ of the same nature.

Choquet-Kendall Theorem (see [19]). Let B be a nonempty convex set spanning a real vector space $X$ and suppose that its minimal affine extension does not contain the zero vector. Let $C$ be the pointed positive convex cone $=\{\lambda b: \lambda \geqslant 0, b \in B\}$ having $B$ as a base (of $X$ ). Then $X$, partly ordered by $C$, will be a vector lattice if and only if both

$\left(1^{\circ}\right) B$ is a simplex,

$\left(2^{\circ}\right)$ each $B$-segment $\{\lambda a+(1-\lambda) b: 0 \leq \lambda \leq 1 ; a, b \in B\}$ is contained in a maximal B-segment. 
Now by applying the Choquet-Kendall theorem, we prove the following theorem in $\mathbb{R}^{n}$.

Theorem 9. Let $B$ be a nonempty convex set spanning $\mathbb{R}^{n}$, for some positive integer $n$, and suppose that its minimal affine extension does not contain the zero vector. Let $C=\{\lambda b: \lambda \geqslant$ $0, b \in B\}$ be the pointed positive convex cone, which has $B$ as its base. Then $\left(\mathbb{R}^{n}, \succcurlyeq_{C}\right)$ is a Hilbert lattice if and only if, in addition to conditions $\left(1^{\circ}\right)$ and $\left(2^{\circ}\right)$ in the Choquet-Kendall theorem, the following condition also holds:

$$
\langle a, b\rangle \geqslant 0, \quad \forall a, b \in C .
$$

Proof. Note that the partial order $\succcurlyeq_{C}$ on $\mathbb{R}^{n}$ induced by the cone $C$ always satisfies the first two conditions of Hilbert lattices (b1) and (b2) recalled in Section 2. From the ChoquetKendall theorem, we only need to show that

$$
\begin{array}{ll}
\langle a, b\rangle \geqslant 0, \quad \forall a, b \in C, \text { if and only if } 0 \preccurlyeq_{C} x \preccurlyeq_{C} y \\
\text { implies }\|x\| \leq\|y\|, \text { for every } x, y \in \mathbb{R}^{n} .
\end{array}
$$

Now we just prove that previous statement.

" $\Longrightarrow$ " Suppose that the inequality $\langle a, b\rangle \geqslant 0$ holds for all $a, b \in C$. Then, for every $x, y \in \mathbb{R}^{n}$ with $0 \preccurlyeq_{C} x \preccurlyeq_{C} y$, we have $y-x \in C$ and $x \in C$. It implies

$$
\begin{aligned}
\langle y, y\rangle & =\langle y-x+x, y-x+x\rangle \\
& =\langle y-x, y-x\rangle+2\langle y-x, x\rangle+\langle x, x\rangle \geqslant\langle x, x\rangle .
\end{aligned}
$$

It implies $\|x\| \leq\|y\|$, which is condition (b3) for the Hilbert lattices.

“ $\Longleftarrow$ Assume that $\left(\mathbb{R}^{n}, \succcurlyeq_{C}\right)$ is a Hilbert lattice satisfying that $0 \preccurlyeq_{C} x \preccurlyeq_{C} y$ implies $\|x\| \leq\|y\|$, for every $x, y \in \mathbb{R}^{n}$. Then, for any $a, b \in C$, we have

$$
a+b \succcurlyeq_{C} a-b, \quad a+b \succcurlyeq_{C} b-a .
$$

Since $|a-b|=(a-b) \vee(b-a)$, the previous order inequalities imply

$$
a+b \succcurlyeq_{C}|a-b|\left(\succcurlyeq_{C} 0\right) .
$$

Then from condition (b3) of the Hilbert lattices recalled in Section 2, we have $\|a+b\| \geqslant\|a-b\|$. That is,

$$
\langle a+b, a+b\rangle \geqslant\langle a-b, a-b\rangle .
$$

Calculating the inner products in $\mathbb{R}^{n}$, it yields $4\langle a, b\rangle \geqslant 0$. It completes the proof.

The following example shows that condition $\left(3^{\circ}\right)$ in Theorem 9 is necessary for $\left(\mathbb{R}^{n}, \succcurlyeq_{C}\right)$ to be a Hilbert lattice.

Example 10. Let $B$ be the closed segment in $\mathbb{R}^{2}$ with ending points at $(1,0)$ and $(-1,1)$. One can show that $B$ is a simplex in $\mathbb{R}^{2}$ under Choquet's definition (9). Then the closed pointed convex cone $C$ generated by $B$ is the closed convex cone in $\mathbb{R}^{2}$ bounded by the two rays with origin as the ending point and passing through points $(1,0)$ and $(-1,1)$, respectively. It can be seen that $B$ is a convex subset spanning $\mathbb{R}^{2}$ and satisfies the two conditions in the Choquet-Kendall theorem. Hence $\mathbb{R}^{2}$, partly ordered by $C$, is a vector lattice (Riesz space). Take two vectors $a, b$ in $C$ with origin as the initial point and with ending points at $(1,0)$ and $(-1,1)$, respectively. It is clear that $\langle a, b\rangle=-1<0$. So $B$ does not satisfy condition $\left(3^{\circ}\right)$ in Theorem 9 , and hence $\left(\mathbb{R}^{2}, \succcurlyeq_{C}\right)$ is not a Hilbert lattice. To more precisely show that $\left(\mathbb{R}^{2}, \succcurlyeq_{C}\right)$ is not a Hilbert lattice, we can show that $\succcurlyeq_{C}$ does not satisfy condition (b3) of the Banach lattices recalled in Section 2. To this end, take $c$ to be the vector in $\mathbb{R}^{2}$ with origin as the initial point and with ending points at $(0,1)$ (in fact, $c \in C$ ). We have $c-b=a \in C$. It yields that $c \geqslant_{C} b \succcurlyeq_{C} 0$. On the other hand, we see that $\|c\|=1$ and $\|b\|=\sqrt{2}$. Hence, the vectors $c$ and $b$ do not satisfy condition (b3) for the Hilbert lattices.

To summarize, we state the connections between vector variational inequalities and ordered variational inequalities as a proposition.

Proposition 11. If $\left(\mathbb{R}^{n}, \succcurlyeq_{C}\right)$ is a Hilbert lattice, that is, the convex cone $C$ in $\mathbb{R}^{n}$ is generated by a simplex $B$ which spans $\mathbb{R}^{n}$ and satisfies the three conditions in Theorem 9, then the ordered variational inequalities (5) and (4) in the Hilbert lattice $\left(\mathbb{R}^{n}, \succcurlyeq_{C}\right)$ coincide with vector variational inequalities (2) and (3), respectively.

\section{Existence of Solutions of Ordered Variational Inequalities on the Banach Lattices}

The convexity and concavity of functions play important roles in nonlinear analysis. In this section, we extend these concepts to order-convexity and order-concavity of mappings in Banach lattices, which will be applied in the proofs of the existence of solutions of ordered variational inequalities in the Banach lattices.

Definition 12. Let $X$ be a Banach space and let $\left(U ; \geqslant^{U}\right)$ be a Banach lattice. Let $C$ be a nonempty convex subset of $X$. A mapping $F: C \rightarrow U$ is said to be

(1) order-convex on $C$ if, for every pair of points $x, y \in C$, the following order inequality holds:

$$
\begin{array}{r}
F(t x+(1-t) y) \preccurlyeq^{U} t F(x)+(1-t) F(y), \\
\text { for every } t \in[0,1],
\end{array}
$$

(2) order-concave on $C$ if, for every pair of points $x, y \in$ $C$, the following order inequality holds:

$$
\begin{array}{r}
F(t x+(1-t) y) \geqslant t F(x)+(1-t) F(y), \\
\text { for every } t \in[0,1] .
\end{array}
$$

The following result is the main theorem of this paper. 
Theorem 13. Let $X$ be a Banach space and let $\left(U ; \geqslant^{U}\right)$ be a Banach lattice. Let $C$ be a nonempty compact convex subset of $X$. Let $F: C \rightarrow L(X, U)$ be a continuous (with respect to the norms) mapping. If $F(x): C \rightarrow U$ has a lower order closed range, that is, $\bigwedge_{y \in C} F(x)(y)$ exists and satisfies

$$
\bigwedge_{y \in C} F(x)(y) \in F(x)(C), \quad \text { for every } x \in C,
$$

then the problem $\operatorname{VOI}(C, F, U)$ has a solution.

Proof. Define a set valued mapping $\Gamma: C \rightarrow 2^{C} \backslash\{\phi\}$ by

$$
\Gamma(x)=\left\{z \in C: F(x) z=\bigwedge_{y \in C} F(x) y\right\},
$$

for any $x \in C$.

The lower order closeness of the ranges of the mapping $F$ in this theorem implies that $\Gamma(x) \neq \phi$, for any $x \in C$. Next, we show that $\Gamma(x)$ is closed for any $x \in C$. To this end, take any $\left\{z_{n}\right\} \subseteq \Gamma(x)$ with $z_{n} \rightarrow z$ in $X$, as $n \rightarrow \infty$. Since $C$ is compact, then $z \in C$. For every fixed $y \in C$, from $z_{n} \in \Gamma(x)$, this yields $F(x)\left(z_{n}\right) \leqslant^{U} F(x)(y)$ that is; $F(x)(y)-F(x)\left(z_{n}\right) \geqslant 0$. It is equivalent to $F(x)(y)-F(x)\left(z_{n}\right) \in U^{+}$, for all $n$. Since $F(x) \in L(X, U)$, then $F(x)$ is a linear and continuous mapping from $X$ to $U$. From $z_{n} \rightarrow z$ in $X$, this implies $F(x)(y)-F(x)\left(z_{n}\right) \rightarrow F(x)(y)-F(x)(z)$, as $n \rightarrow \infty$. From Theorem 1 recalled in Section $2, U^{+}$is norm closed. It implies that $F(x)(y)-F(x)(z) \in U^{+}$. That is, $F(x)(z) \aleph^{U} F(x)(y)$, for any given $y \in C$. Noticing that $z \in C$, we obtain $F(x)(z)=$ $\bigwedge_{y \in C} F(x) y$. Hence $z \in \Gamma(x)$ and therefore $\Gamma(x)$ is closed.

To show that $\Gamma(x)$ is convex, take any $z_{1}, z_{2} \in \Gamma(x)$ and for any $t \in[0,1]$ from the linearity of $F(x)$ and applying the linearity and transitive property of the lattice order $\geqslant U$ in the Banach lattice $\left(U ; \geqslant^{U}\right)$, we have

$$
\begin{aligned}
F(x) & \left(t z_{1}+(1-t) z_{2}\right) \\
= & t F(x)\left(z_{1}\right)+(1-t) F(x)\left(z_{2}\right) \\
& =t \bigwedge_{y \in C} F(x) y+(1-t) \bigwedge_{y \in C} F(x) y \\
& =\bigwedge_{y \in C} F(x) y .
\end{aligned}
$$

Since $C$ is convex, so $t z_{1}+(1-t) z_{2} \in C$. The previous order inequalities imply $t z_{1}+(1-t) z_{2} \in \Gamma(x)$. Hence, $\Gamma(x)$ is convex.

Next, we prove that the mapping $\Gamma$ is a upper semicontinuous set valued mapping. Since $C$ is compact, so $\Gamma$ is a set valued mapping from a compact topological space $C$ to $C$. From Theorem 7 recalled in Section 2, it is sufficient to prove that the following set is closed:

$$
\operatorname{graph} \Gamma=\{(u, v) \in C \times C: v \in \Gamma(u)\} .
$$

For this purpose, take any sequence $\left\{\left(u_{n}, v_{n}\right)\right\} \subseteq$ graph $\Gamma$ with $u_{n} \rightarrow u$ and $v_{n} \rightarrow v$ in $X$, as $n \rightarrow \infty$. We have

$$
F\left(u_{n}\right)\left(v_{n}\right)=\bigwedge_{y \in C} F\left(u_{n}\right)(y), \quad \text { for } n=1,2,3, \ldots
$$

Since $C$ is compact, then $u \in C$. For every fixed $y \in C$, from $v_{n} \in \Gamma\left(u_{n}\right)$, this yields $F\left(u_{n}\right)\left(v_{n}\right) \aleph^{U} F\left(u_{n}\right)(y)$; that is, $F\left(u_{n}\right)(y)-F\left(u_{n}\right)\left(v_{n}\right) \geqslant 0$. Thus, $F\left(u_{n}\right)(y)-F\left(u_{n}\right)\left(v_{n}\right) \in U^{+}$, for all $n$. From the continuity condition of $F: C \rightarrow L(X, U)$, since $u_{n} \rightarrow u$ in $X$, as $n \rightarrow \infty$, we have

$$
F\left(u_{n}\right) \longrightarrow F(u) \text { in } L(X, U), \quad \text { as } n \longrightarrow \infty .
$$

From $v_{n} \rightarrow v$ in $X$, as $n \rightarrow \infty$, and $F\left(u_{n}\right), F(u) \in L(X, U)$, for all $n$, the previous limit implies

$$
\begin{array}{r}
F\left(u_{n}\right)(y)-F\left(u_{n}\right)\left(v_{n}\right) \longrightarrow F(u)(y)-F(u)(v) \text { in } U, \\
\text { as } n \longrightarrow \infty
\end{array}
$$

Since $U^{+}$is norm closed and $F\left(u_{n}\right)(y)-F\left(u_{n}\right)\left(v_{n}\right) \in U^{+}$, for all $n$, this implies $F(u)(y)-F(u)(v) \in U^{+}$. That is, $F(u)(v) \preccurlyeq^{U} F(u)(y)$, for any given $y \in C$. The compactness of $C$ and $v_{n} \rightarrow v$ in $X$, as $n \rightarrow \infty$, and $v_{n} \in C$ imply that $v \in C$. Then from $F(u)(v)=\bigwedge_{y \in C} F(u)(y)$, we get $v \in \Gamma(u)$. We obtain that $(u, v) \in \operatorname{graph} \Gamma$, and hence graph $\Gamma$ is closed.

To summarize, we obtain that $\Gamma$ is an upper semicontinuous set valued mapping with nonempty compact convex values. From the Bohnenblust-Karlin fixed point theorem, the set of fixed points of $\Gamma$ is nonempty. Taking a fixed point $x^{*}$ of $V$, we have $x^{*} \in \Gamma\left(x^{*}\right)$. That is,

$$
F\left(x^{*}\right)\left(x^{*}\right)=\bigwedge_{y \in C} F\left(x^{*}\right)(y) .
$$

This implies

$$
F\left(x^{*}\right)\left(x^{*}\right) \preccurlyeq^{U} F\left(x^{*}\right)(y), \quad \forall y \in C .
$$

Noticing that $F\left(x^{*}\right)$ is linear, from the properties of the lattice order $\preccurlyeq^{U}$, it is equivalent to

$$
F\left(x^{*}\right)\left(y-x^{*}\right) \geqslant 0, \quad \forall y \in C .
$$

Hence $x^{*}$ is a solution to the ordered variational inequality problem $\operatorname{VOI}(C, F, U)$. This theorem is proved.

\section{Applications to Order-Optimization Problems}

As some applications of Theorem 13, we solve some orderoptimization problems in the Banach lattices in this section.

Definition 14. Let $X$ be a Banach space and let $\left(U ; \geqslant^{U}\right)$ be a Banach lattice. Let $C$ be a nonempty subset of $X$. Let $F: C \rightarrow$ $L(X, U)$ be a mapping. $F$ is said to take an associated orderminimum value at a point $x^{*} \in C$ if it satisfies

$$
F\left(x^{*}\right)\left(x^{*}\right) \preccurlyeq^{U} F\left(x^{*}\right)(x), \quad \forall x \in C .
$$

$F$ is said to take an associated order-maximum value at a point $x^{*} \in C$ if it satisfies

$$
F\left(x^{*}\right)\left(x^{*}\right) \geqslant F\left(x^{*}\right)(x), \quad \forall x \in C .
$$


From the proof of Theorem 13 and from the linearity of $F(x)$, for all $x \in C$, it is clear that (32) is equivalent to (9). Hence, a point $x^{*} \in C$ is a solution to the problem $\operatorname{VOI}(C, F, U)$ if and only if, at the point $x^{*}, F$ takes an associated order-minimum value. So the following corollary is an immediate consequence of Theorem 13.

Corollary 15. Let $X$ be a Banach space and let $\left(U ; \geqslant^{U}\right)$ be a Banach lattice. Let $C$ be a nonempty compact convex subset of $X$. Let $F: C \rightarrow L(X, U)$ be a continuous (with respect to the norms) mapping. If $F(x): C \rightarrow U$ has a lower order closed range, that is, $\bigwedge_{y \in C} F(x)(y)$ exists and satisfies

$$
\bigwedge_{y \in C} F(x)(y) \in F(x)(C), \quad \text { for every } x \in C,
$$

then there is a point $x^{*} \in C$, at which $F$ takes an associated order-minimum value.

The existence of associated order-maximum value problem is also considered as a consequence of Theorem 13. We provide a solution of this problem as follows as a corollary of Theorem 13.

Corollary 16. Let $X$ be a Banach space and let $\left(U ; \geqslant^{U}\right)$ be a Banach lattice. Let $C$ be a nonempty compact convex subset of $X$. Let $G: C \rightarrow L(X, U)$ be a continuous (with respect to the norms) mapping. If $G(x): C \rightarrow U$ has an upper order closed range, that is, $\bigvee_{y \in C} G(x)(y)$ exists and satisfies

$$
\bigvee_{y \in C} G(x)(y) \in G(x)(C), \quad \text { for every } x \in C,
$$

then there is a point $y^{*} \in C$, at which $G$ takes an associated order-maximum value.

Proof. For the given mapping $G: C \rightarrow L(X, U)$, define $F$ : $C \rightarrow L(X, U)$ by $F(x)=-G(x)$, for every $x \in C$,

Then $F: C \rightarrow L(X, U)$ is also a continuous (with respect to the norms) mapping. For every $x \in C$, we have

$$
\begin{aligned}
\bigwedge_{y \in C} F(x)(y) & =\bigwedge_{y \in C}(-G(x)(y)) \\
& =-\bigvee_{y \in C} G(x)(y) \in-G(x)(C)=F(x)(C) .
\end{aligned}
$$

From Corollary 15, there is a point $y^{*} \in C$, at which $F$ takes an associated order-minimum value; that is,

$$
F\left(y^{*}\right)\left(y^{*}\right) \preccurlyeq^{U} F\left(y^{*}\right)(x), \quad \forall x \in C .
$$

Then we have

$$
-G\left(y^{*}\right)\left(y^{*}\right) \preccurlyeq^{U}-G\left(y^{*}\right)(x), \quad \forall x \in C .
$$

It is equivalent to

$$
G\left(y^{*}\right)\left(y^{*}\right) \geqslant G\left(y^{*}\right)(x), \quad \forall x \in C .
$$

Hence, $G$ takes an associated order-maximum value at the point $y^{*}$.

\section{Acknowledgment}

This research was partially supported by National Natural Science Foundation of China (11171137) and the National Natural Science Foundation of China (Grant 11101379).

\section{References}

[1] G. Isac, Complementarity Problems, vol. 1528 of Lecture Notes in Mathematics, Springer, Berlin, Germany, 1992.

[2] G. Isac and J. Li, "Complementarity problems, Karamardian's condition and a generalization of Harker-Pang condition," Nonlinear Analysis Forum, vol. 6, no. 2, pp. 383-390, 2001.

[3] S. Karamardian, "Complementarity problems over cones with monotone and pseudomonotone maps," Journal of Optimization Theory and Applications, vol. 18, no. 4, pp. 445-454, 1976.

[4] D. Kinderlehrer and G. Stampacchia, An Introduction to Variational Inequalities and Their Applications, vol. 88, Academic Press, New York, NY, USA, 1980.

[5] J. C. Yao, "Variational inequalities with generalized monotone operators," Mathematics of Operations Research, vol. 19, no. 3, pp. 691-705, 1994.

[6] F. Giannessi, "Theorems of alternative, quadratic programs and complementarity problems," in Variational Inequalities and Complementarity Problems, pp. 151-186, Wiley, Chichester, UK, 1980.

[7] F. Giannessi, "Theorems of the alternative and optimality conditions," Journal of Optimization Theory and Applications, vol. 42, no. 3, pp. 331-365, 1984.

[8] F. Giannessi, Constrained Optimization and Image Space Analysis, vol. 1, Springer, New York, NY, USA, 2005.

[9] F. Giannessi, G. Mastroeni, and L. Pellegrini, "On the theory of vector optimization and variational inequalities. Image space analysis and separation," in Vector Variational Inequalities and Vector Equilibria, vol. 38, pp. 153-215, Kluwer Academic Publishers, Dordrecht, The Netherlands, 2000.

[10] N.-J. Huang and Y.-P. Fang, "On vector variational inequalities in reflexive Banach spaces," Journal of Global Optimization, vol. 32, no. 4, pp. 495-505, 2005.

[11] R. P. Agarwal, M. Balaj, and D. O'Regan, "A unifying approach to variational relation problems," Journal of Optimization Theory and Applications, vol. 155, no. 2, pp. 417-429, 2012.

[12] G. Mastroeni and L. Pellegrini, "On the image space analysis for vector variational inequalities," Journal of Industrial and Management Optimization, vol. 1, no. 1, pp. 123-132, 2005.

[13] J. Li and N. J. Huang, "Image space analysis for vector variational inequalities with matrix inequality constraints and applications," Journal of Optimization Theory and Applications, vol. 145, no. 3, pp. 459-477, 2010.

[14] D. T. Luc, Theory of Vector Optimization, vol. 319 of Lecture Notes in Economics and Mathematical Systems, Springer, Berlin, Germany, 1989.

[15] F. Facchinei and J. S. Pang, Finite-Dimensional Variational Inequalities and Complementarity Problems, Springer, New York, NY, USA, 2003.

[16] J. L. Li and C. F. Wen, "Ordered variational inequalities and Order-complementarity Problems in Banach Lattices," Abstract and Applied Analysis, vol. 2013, Article ID 323126, 9 pages, 2013.

[17] C. D. Aliprantis and O. Burkinshaw, Positive Operators, Springer, Dordrecht, The Netherlands, 2006. 
[18] S. Park, "Fixed points, intersection theorems, variational inequalities, and equilibrium theorems," International Journal of Mathematics and Mathematical Sciences, vol. 24, no. 2, pp. 7393, 2000.

[19] D. G. Kendall, "Simplexes and vector lattices," Journal of the London Mathematical Society, vol. 37, pp. 365-371, 1962. 


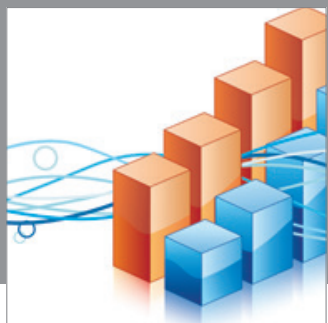

Advances in

Operations Research

mansans

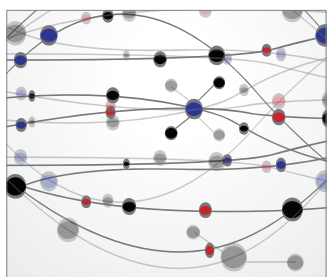

The Scientific World Journal
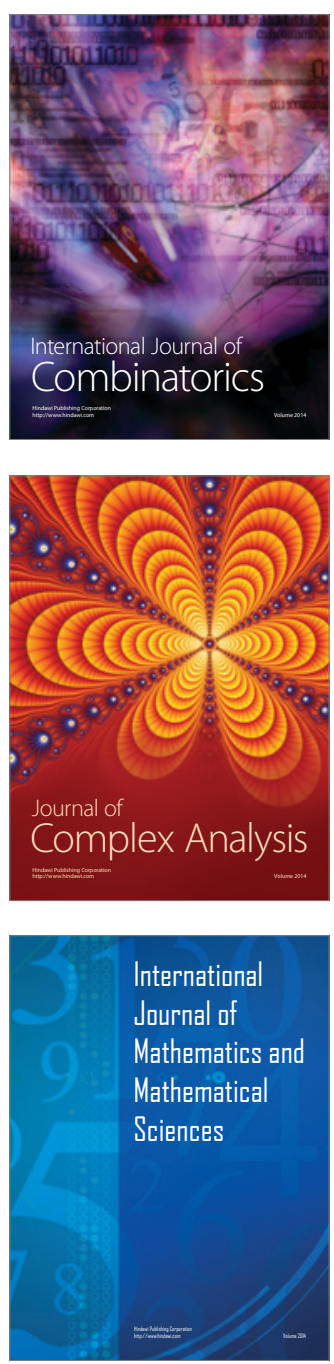
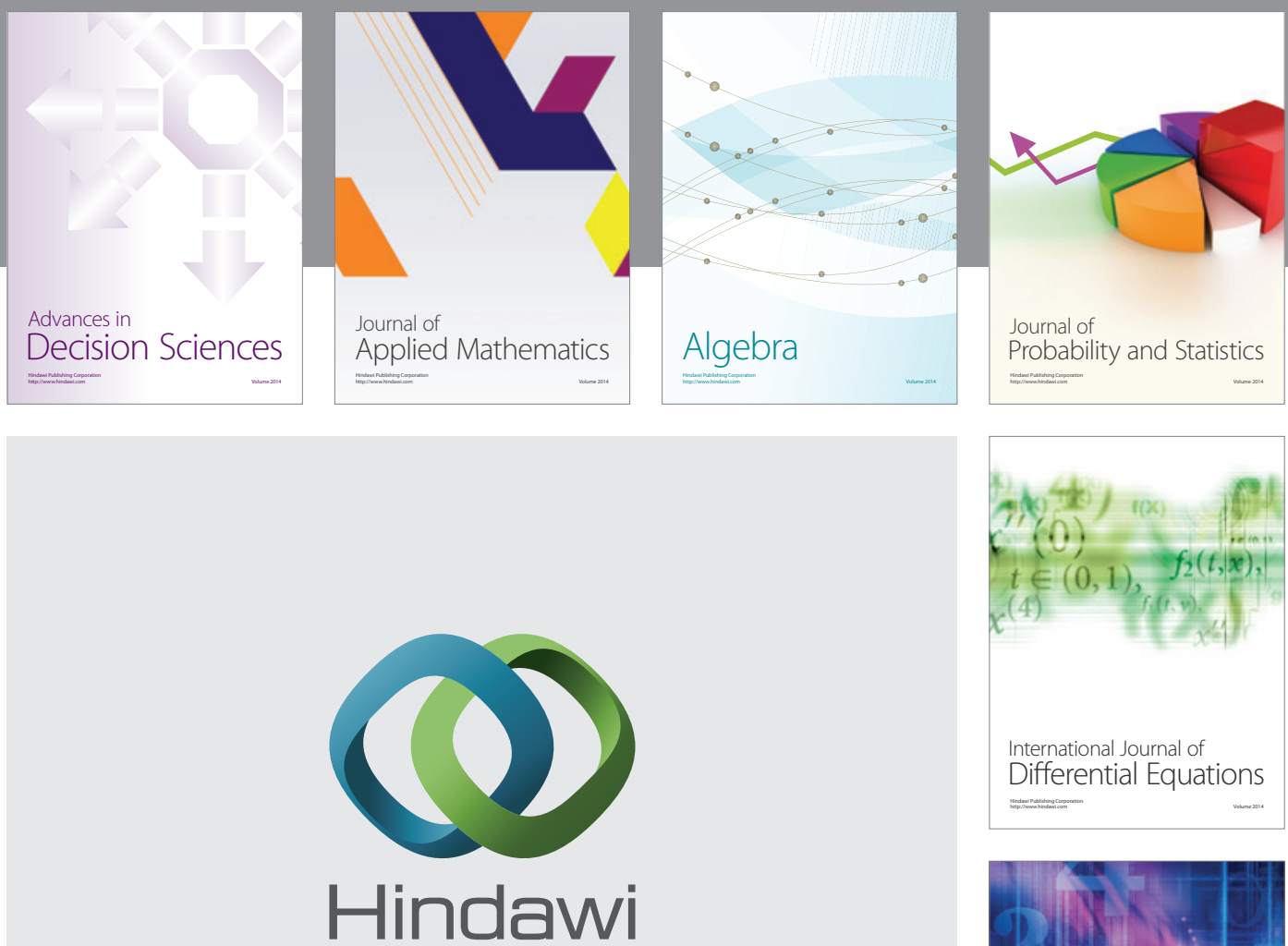

Submit your manuscripts at http://www.hindawi.com
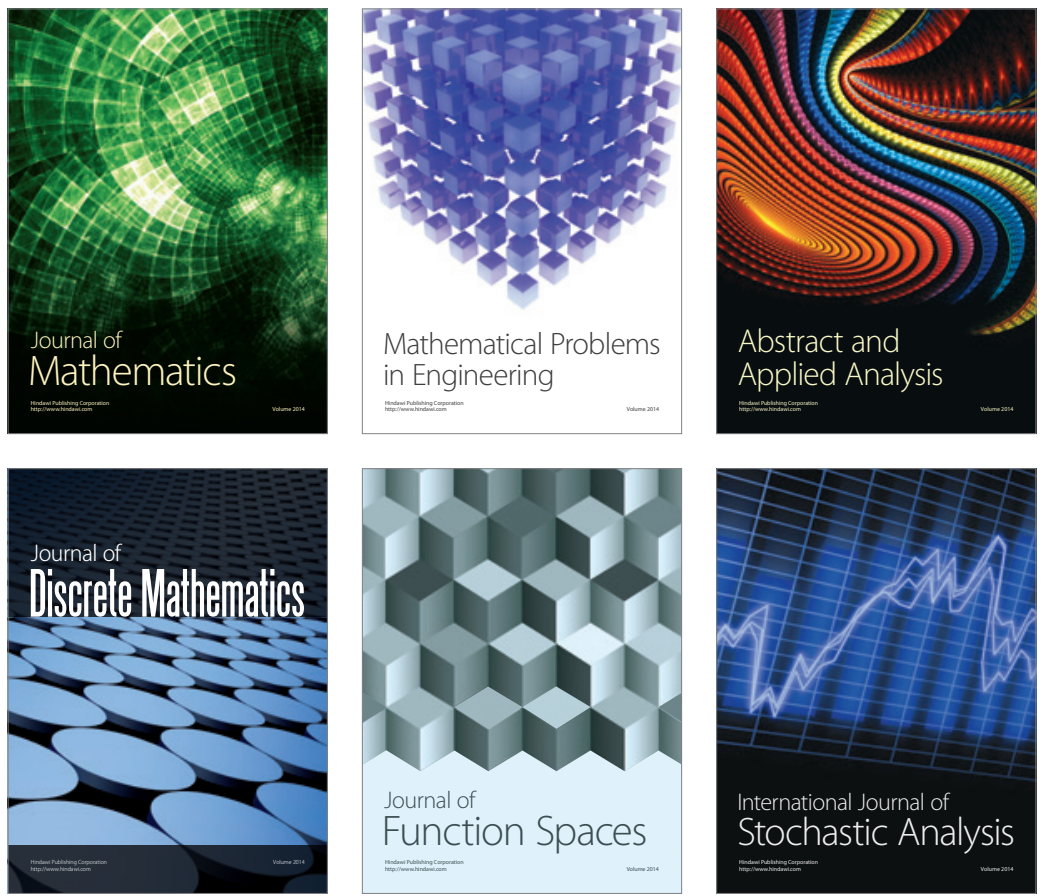

Journal of

Function Spaces

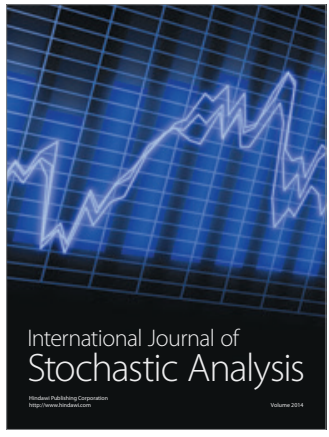

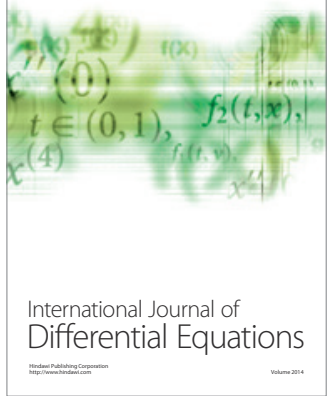
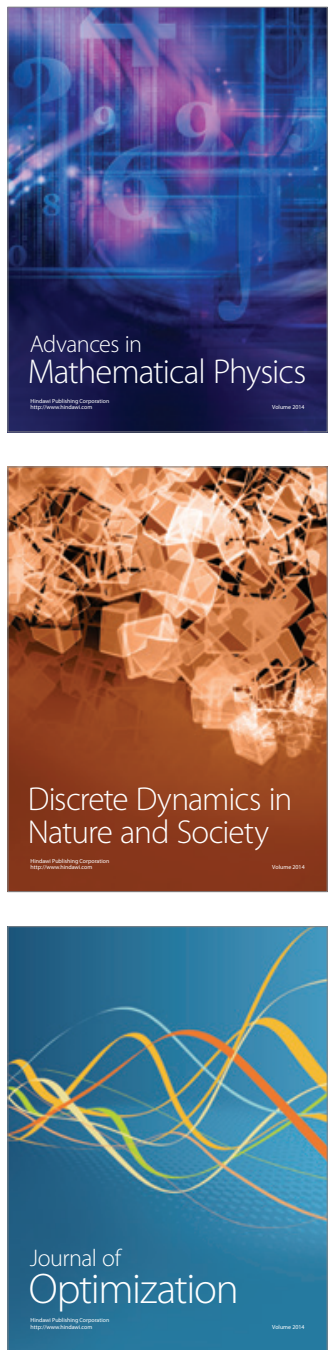